\title{
2-2011
}

\section{Surface Groups Within Baumslag Doubles}

\author{
Benjamin Fine \\ Fairfield University, fine@fairfield.edu \\ Gerhard Rosenberger
}

Follow this and additional works at: https://digitalcommons.fairfield.edu/mathandcomputerscience-

facultypubs

Copyright 2011 by Cambridge University Press. Original published version can be found at DOI: $10.1017 /$ S0013091509001102

\section{Peer Reviewed}

\section{Repository Citation}

Fine, Benjamin and Rosenberger, Gerhard, "Surface Groups Within Baumslag Doubles" (2011).

Mathematics Faculty Publications. 11.

https://digitalcommons.fairfield.edu/mathandcomputerscience-facultypubs/11

\section{Published Citation}

B. Fine, and G. Rosenberger. Surface Groups Within Baumslag Doubles, Proceedings of the Edinburgh Mathematical Society. 54 (Part I), 91-97.

This Article is brought to you for free and open access by the Mathematics Department at DigitalCommons@Fairfield. It has been accepted for inclusion in Mathematics Faculty Publications by an authorized administrator of DigitalCommons@Fairfield. For more information, please contact digitalcommons@fairfield.edu. 


\title{
SURFACE GROUPS WITHIN BAUMSLAG DOUBLES
}

\author{
BENJAMIN FINE ${ }^{1}$ AND GERHARD ROSENBERGER ${ }^{2}$ \\ ${ }^{1}$ Department of Mathematics, Fairfield University, \\ Fairfield, CT 06430, USA (fine@fairfield.edu) \\ ${ }^{2}$ Heinrich-Barth Strasse 1, 20146 Hamburg, Germany
}

(Received 7 August 2009)

Dedicated to Colin Maclachlan on the occasion of his 70th birthday

\begin{abstract}
A conjecture of Gromov states that a one-ended word-hyperbolic group must contain a subgroup that is isomorphic to the fundamental group of a closed hyperbolic surface. Recent papers by Gordon and Wilton and by Kim and Wilton give sufficient conditions for hyperbolic surface groups to be embedded in a hyperbolic Baumslag double $G$. Using Nielsen cancellation methods based on techniques from previous work by the second author, we prove that a hyperbolic orientable surface group of genus 2 is embedded in a hyperbolic Baumslag double if and only if the amalgamated word $W$ is a commutator: that is, $W=[U, V]$ for some elements $U, V \in F$. Furthermore, a hyperbolic Baumslag double $G$ contains a non-orientable surface group of genus 4 if and only if $W=X^{2} Y^{2}$ for some $X, Y \in F$. G can contain no non-orientable surface group of smaller genus.
\end{abstract}

Keywords: hyperbolic group; orientable surface group; quadratic word

2010 Mathematics subject classification: Primary 20F67

Secondary 20F65; 20E06; 20E07

\section{Introduction}

A Baumslag double is an amalgamated product of the form

$$
G=F_{\{W=\bar{W}\}}^{\star} \bar{F},
$$

where $F$ is a finitely generated free group, $\bar{F}$ is an isomorphic copy, $W$ is a non-trivial word in $F$ and $\bar{W}$ is its copy in $\bar{F}$. An orientable surface group of genus 2 is a Baumslag double and, in fact, Baumslag doubles were introduced in $[\mathbf{2}]$ to prove that surface groups are residually free. If $G$ is a Baumslag double and if the identified word $W$ is not a proper power in $F$, it follows from the combination theorems of Juhasz and Rosenberger [7], Kharlampovich and Myasnikov [8] and Bestvina and Feighn [3] that the group $G$ is hyperbolic. In fact, the Baumslag double $G$ is hyperbolic if and only if $W$ is not a proper power in $F$ because $W$ is a proper power in $F$ if and only if $\bar{W}$ is a proper power in $\bar{F}$.

An open conjecture of Gromov [6] states that a one-ended word-hyperbolic group must contain a subgroup that is isomorphic to the fundamental group of a closed hyperbolic 
surface. Recent work by Gordon and Wilton [6] and by Kim and Wilton [9] gives sufficient conditions for hyperbolic surface groups to be embedded in a Baumslag double $G$. The work of Gordon and Wilton uses group cohomology and 3-manifold theory, while that of Kim and Wilton proceeds by realizing a Baumslag double as the fundamental group of a non-positively curved square complex.

In this paper, we use Nielsen cancellation methods based on the techniques in [12] to prove that a hyperbolic orientable surface group of genus 2 is embedded in a hyperbolic Baumslag double if and only if the amalgamated word $W$ is a commutator: that is, $W=[U, V]$ for some elements $U, V \in F$. Since an orientable surface group of genus 2 contains surface groups of all finite genus, it follows that $G$ contains hyperbolic surface groups of all finite genus if and only if $W$ is a commutator in $F$. Furthermore, a Baumslag double $G$ contains a non-orientable surface group of genus 4 if and only if $W=X^{2} Y^{2}$ for some $X, Y \in F$.

\section{Main result}

As mentioned in $\S 1$ it follows from the combination theorems of Juhasz and Rosenberger [7], Kharlampovich and Myasnikov [8] and Bestvina and Feighn [3] that the Baumslag double

$$
G=F \underset{\{W \stackrel{\star}{=} \bar{W}\}}{\star} \bar{F}
$$

is hyperbolic if and only if the identified word $W$ is not a proper power in $F$. We call such a group a hyperbolic Baumslag double. Here we assume that $W$ is a reduced word in the free group $F$. Our main result is the following.

Theorem 2.1. Let

$$
G=F \underset{\{W \stackrel{\star}{=} \bar{W}\}}{\star} \bar{F}
$$

be a hyperbolic Baumslag double. Then $G$ contains a hyperbolic orientable surface group of genus 2 if and only if $W$ is a commutator: that is, $W=[U, V]$ for some elements $U, V \in F$. Furthermore, a Baumslag double $G$ contains a non-orientable surface group of genus 4 if and only if $W=X^{2} Y^{2}$ for some $X, Y \in F$.

Since an orientable surface group of genus 2 contains an orientable surface group of any finite genus as a subgroup, we immediately get the following corollary.

Corollary 2.2. Let

$$
G=F_{\{W=\bar{W}\}}^{\star} \bar{F}
$$

be a hyperbolic Baumslag double. Then $G$ contains orientable surface groups of all finite genus if and only if $W$ is a commutator.

Before giving the proof we recall some material about surface groups and cyclically pinched one-relator groups.

A surface group is the fundamental group of a compact orientable or non-orientable surface. If the genus of the surface is $g$, then we say that the corresponding surface group also has genus $g$. 
An orientable surface group $S_{g}$ of genus $g \geqslant 1$ has a one-relator presentation of the form

$$
S_{g}=\left\langle a_{1}, b_{1}, \ldots, a_{g}, b_{g} ;\left[a_{1}, b_{1}\right] \cdots\left[a_{g}, b_{g}\right]=1\right\rangle,
$$

while a non-orientable surface group $T_{g}$ of genus $g \geqslant 1$ also has a one-relator presentation, which now has the form

$$
T_{g}=\left\langle a_{1}, a_{2}, \ldots, a_{g} ; a_{1}^{2} a_{2}^{2} \cdots a_{g}^{2}=1\right\rangle .
$$

Much of combinatorial group theory originally arose out of the theory of one-relator groups and the concepts and ideas surrounding the Freiheitssatz or Independence Theorem of Magnus (see [11] or [10]). Going backwards, the ideas of the Freiheitssatz were motivated by the topological properties of surface groups $[\mathbf{1}]$.

The algebraic generalization of the one-relator presentation type of a surface group presentation leads to cyclically pinched one-relator groups. These groups have the same general form as a surface group and have proved to be quite amenable to study. In particular, a cyclically pinched one-relator group is a one-relator group of the following form:

$$
G=\left\langle a_{1}, \ldots, a_{p}, a_{p+1}, \ldots, a_{n} ; U=V\right\rangle,
$$

where $1 \neq U=U\left(a_{1}, \ldots, a_{p}\right)$ is a cyclically reduced, non-primitive (i.e. not part of a free basis) word in the free group $F_{1}$ on $a_{1}, \ldots, a_{p}$ and where $1 \neq V=V\left(a_{p+1}, \ldots, a_{n}\right)$ is a cyclically reduced, non-primitive word in the free group $F_{2}$ on $a_{p+1}, \ldots, a_{n}$.

Clearly, such a group is the free product of the free groups on $a_{1}, \ldots, a_{p}$ and $a_{p+1}, \ldots, a_{n}$, respectively, amalgamated over the cyclic subgroups generated by $U$ and $V$. Cyclically pinched one-relator groups have been shown to be extremely similar to surface groups $[\mathbf{1}]$.

A cyclically pinched one-relator group is hyperbolic if either $U$ or $V$ is not a proper power in its respective free group factor $[\mathbf{3}, \mathbf{7}, \mathbf{8}]$.

In [2], Baumslag introduced a double of a free group, now called a Baumslag double, in order to prove that orientable surface groups are residually free. In that paper he also proved that if neither $U$ nor $V$ is a proper power, then a cyclically pinched one-relator group is 2-free: that is, any 2-generator subgroup must be free. This was generalized by Rosenberger, who proved the following result [12, Theorem 3.3, p. 335] using Nielsen cancellation methods. This result is one of the bases for the proof of Theorem 2.1.

Theorem 2.3 (Rosenberger [12]). Let $G$ be a cyclically pinched one-relator group of the form

$$
G=\left\langle a_{1}, \ldots, a_{p}, a_{p+1}, \ldots, a_{n} ; W=V\right\rangle,
$$

where $1 \neq W=W\left(a_{1}, \ldots, a_{p}\right)$ is a cyclically reduced, non-primitive (i.e. not part of a free basis) word in the free group $F_{1}$ on $a_{1}, \ldots, a_{p}$ and where $1 \neq V=V\left(a_{p+1}, \ldots, a_{n}\right)$ is a cyclically reduced, non-primitive word in the free group $F_{2}$ on $a_{p+1}, \ldots, a_{n}$. Suppose that neither $W$ nor $V$ is a proper power in its respective free group factor. Then we have the following. 
(a) Every subgroup $H \subset G$ of rank 3 is free of rank 3 .

(b) Let $H \subset G$ be a subgroup of rank 4 . One of the following two cases then occurs.

(i) $H$ is free of rank 4.

(ii) If $\left\{x_{1}, x_{2}, x_{3}, x_{4}\right\}$ is a generating system of $H$, then there is a Nielsen transformation from $\left\{x_{1}, x_{2}, x_{3}, x_{4}\right\}$ to a system $\left\{y_{1}, \ldots, y_{n}\right\}$ with $y_{1}, y_{2} \in z F_{1} z^{-1}$ and $y_{3}, y_{4} \in z F_{2} z^{-1}$ for some $z \in G$. Moreover, there is a one-relator presentation for $H$ on the generating system $\left\{x_{1}, x_{2}, x_{3}, x_{4}\right\}$.

Before presenting the proof we need two other ideas concerning Nielsen cancellation in free products with amalgamation. A word $w \in F$, where $F$ is a free group on $x_{1}, \ldots, x_{n}$, is regular if there exists no automorphism $\alpha: F \mapsto F$ such that $\alpha(w)=w^{\prime}$, when written as a word in $x_{1}, \ldots, x_{n}$, contains fewer of the generators than $w$ itself does. An ordered set $U=\left\{u_{1}, \ldots, u_{n}\right\} \subset F$ is regular if there exists no Nielsen transformation from $U$ to a system $U^{\prime}=\left\{u_{1}^{\prime}, \ldots, u_{n}^{\prime}\right\}$ in which one of the elements equals 1 . This type of regularity is extended to free products with amalgamation in the following way. Suppose that $G$ is a free product with amalgamation with factors $H_{1}$ and $H_{2}$ such that $G=H_{1} \star_{A} H_{2}$. An ordered set $U=\left\{u_{1}, \ldots, u_{n}\right\} \subset G$ is then regular if there exists no Nielsen transformation from $U$ to a system $U^{\prime}=\left\{u_{1}^{\prime}, \ldots, u_{n}^{\prime}\right\}$ in which one of the elements is conjugate to an element of $A$.

The other crucial result for the proof of Theorem 2.1 is the following technical theorem [5, Theorem 5.3]. Recall that if $F$ is a free group on $X=\left\{x_{1}, \ldots, x_{n}\right\}$, then a reduced word $w=w\left(x_{1}, \ldots, x_{n}\right)$ is a quadratic word if each $x_{i}$, which appears in $w$ as $x_{i}$ or $x_{i}^{-1}$, appears exactly twice. For example, the surface group word of genus $2,\left[x_{1}, x_{2}\right]\left[x_{3}, x_{4}\right]=$ $x_{1}^{-1} x_{2}^{-1} x_{1} x_{2} x_{3}^{-1} x_{4}^{-1} x_{3} x_{4}$, is a quadratic word.

Theorem 2.4 (Fine et al. [5]). Suppose that $G=H_{1} \star_{A} H_{2}$ with $H_{1} \neq A \neq H_{2}$ and $A$ malnormal in both $H_{1}$ and $H_{2}$. Let $F$ be a free group of rank $n$ with $1 \leqslant n \leqslant 4$ and let $1 \neq w=w\left(x_{1}, \ldots, x_{n}\right)$ be a regular quadratic word on the ordered basis $X=$ $\left\{x_{1}, \ldots, x_{n}\right\}$. Furthermore, let $\phi: F \mapsto G$ be a homomorphism such that $U=\phi(X)$ is regular in $G$ and $\phi(w)=1$. Then the pair $(w, U)$ is Nielsen equivalent to a pair $\left(w^{\prime}, U^{\prime}\right)=\left(\alpha(w), \alpha^{-1}(U)\right)$ with $\alpha: F \mapsto F$ an automorphism such that

(1) $w^{\prime}=w_{1} w_{2}$, where $w_{1}, w_{2}$ are also quadratic in $F$,

(2) for $i=1,2$ we have that $\phi\left(\alpha^{-1}\left(w_{i}\right)\right)$ is conjugate to an element of $A$ and

(3) for $i=1,2$ there is a $\nu_{i} \in\{1,2\}$ and a $g_{i} \in G$ with $\phi\left(\alpha^{-1}\left(x_{j}\right)\right) \in g_{i} H_{\nu_{i}} g_{i}^{-1}$ for each $x_{j}$ that occurs in $w_{i}$.

We now give the proof of Theorem 2.1.

Proof of Theorem 2.1. Suppose that

$$
G=F \underset{\{W \stackrel{\star}{=} \bar{W}\}}{ } \bar{F}
$$


is a hyperbolic Baumslag double, where $F$ is a free group on $X=\left\{x_{1}, \ldots, x_{n}\right\}$. Since we are assuming hyperbolicity, we have that $W$ and hence $\bar{W}$ are not proper powers. Furthermore, if $W$ were either trivial or primitive in $F$, then $G$ would be a free group, so $G$ could not contain a surface group that is either orientable or non-orientable. Furthermore, if $G$ contains a surface group, then $G$ cannot be a free group and hence $W$ is neither trivial nor primitive. Therefore, we may assume that the amalgamated word $W$ is neither trivial nor primitive.

We consider the orientable case first. Suppose that $W=[u, v]$ in $F$. Then

$$
W(\bar{W})^{-1}=[u, v]([\bar{u}, \bar{v}])^{-1}=[u, v][\bar{v}, \bar{u}]=1 .
$$

Consider the subgroup $H=\langle u, v, \bar{u}, \bar{v}\rangle$ of $G$. We can see that $H$ cannot be a free group by applying Theorems 2.3 and 2.4 to the equation $[u, v][\bar{u}, \bar{v}]=1$ in $G$ and from the fact that $G$ does not contain a free abelian group of rank 2 . Hence $H$ is a one-relator group of rank 4 by Theorem 2.3. We show that a defining relation is precisely $[u, v][\bar{v}, \bar{u}]=1$.

Let $G$ be a cyclically pinched one-relator group of the form

$$
G=\left\langle a_{1}, \ldots, a_{p}, a_{p+1}, \ldots, a_{n} ; W=V\right\rangle
$$

as in Theorem 2.3. Let $H=\left\langle x_{1}, x_{2}, x_{3}, x_{4}\right\rangle$ be a rank 4 subgroup of $G$. Within the proof of Theorem 2.3 [12, Theorem 3.3, pp. 335-340] it is shown that if $H$ is not free, then not only is $H$ a one-relator group but a method is described showing how to obtain a defining relation for $H[\mathbf{1 2}$, p. 340]. This is done in the following manner. If there is a Nielsen transformation from $\left\{x_{1}, x_{2}, x_{3}, x_{4}\right\}$ to a system where one element is conjugate to an element in the amalgamated subgroup, then $H$ is free of rank 4 . Now assume that $H$ is not free of rank 4. Then, by the statement of Theorem 2.3, $G$ is a one-relator group, and we may assume, possibly after a Nielsen transformation and a conjugation, that $x_{1}, x_{2}$ are in $F_{1}$, the free group on $a_{1}, \ldots, a_{p}$, that $x_{3}, x_{4}$ are in $F_{2}$, the free group on $a_{p+1}, \ldots, a_{n}$, and that $W$ is in $\left\langle x_{1}, x_{2}\right\rangle$ or $V$ is in $\left\langle x_{3}, x_{4}\right\rangle$. Let $W$ be in $\left\langle x_{1}, x_{2}\right\rangle$. We consider the subgroup $K=\left\langle V, x_{3}, x_{4}\right\rangle$ in $F_{2}$. (Recall that $W=V$ in $G$.) $K$ cannot be a free group of rank 3 because otherwise $H$ is free of rank 4 . Hence $K$ is a one-relator group in $V, x_{3}, x_{4}$ and therefore $H$ is a one-relator group in $x_{1}, x_{2}, x_{3}, x_{4}$. The relation is obtained as follows. Take the relation for $K$ and replace $V$ by $W$ as a word in $x_{1}, x_{2}$.

If we apply this to the hyperbolic Baumslag double with $W=[u, v]$, we must consider the situation where we have a free group $F=\langle a, b ;\rangle$ of rank 2 generated by a system $\{r, s,[a, b]\}$. However, if $F=\langle a, b ;\rangle$ is generated by a system $\{r, s,[a, b]\}$, it follows from [12, Lemma 3.17] (see also [13, Hilfsatz 5]) that there is a free Nielsen transformation $\mathcal{T}$ from $\{r, s,[a, b]\}$ to $\{a, b,[a, b]\}$, where $[a, b]$ is not replaced. Not replaced means that in all the elementary Nielsen transformations of which $\mathcal{T}$ is composed, the commutator $[a, b]$ either remains unchanged, is changed to $([a, b])^{-1}$ or is put in a different location in the respective triple (see [12, pp. 335-340] for more details). In the hyperbolic Baumslag double the transformations are identical in the other factor. Therefore, $[u, v]=[\bar{u}, \bar{v}]$ must be a defining relation for $H$. It follows that $H$ is an orientable surface group of genus 2 and $G$ contains such a subgroup. 
Conversely, let $H$ be a subgroup of $G$ that is an orientable surface group of genus 2 . Hence $H$ has a presentation

$$
H=\left\langle x_{1}, x_{2}, x_{3}, x_{4} ;\left[x_{1}, x_{2}\right]\left[x_{3}, x_{4}\right]=1\right\rangle .
$$

Consider the system $\left\{x_{1}, x_{2}, x_{3}, x_{4}\right\} \subset G$ and apply Nielsen cancellations within the amalgamated free product $G$ with respect to the quadratic word $v=\left[x_{1}, x_{2}\right]\left[x_{3}, x_{4}\right]$.

The system $\left\{x_{1}, x_{2}, x_{3}, x_{4}\right\}$ is regular. That is, there is no Nielsen transformation from $\left\{x_{1}, x_{2}, x_{3}, x_{4}\right\}$ to a system that contains an element that is conjugate in $G$ to a power of $W$ or $\bar{W}$. If the system $\left\{x_{1}, \ldots, x_{n}\right\}$ was not regular, then $H$ would have to be a free group from [12, Lemma 3.1]. Now we apply Theorem 2.4 to $X=\left\{x_{1}, x_{2}, x_{3}, x_{4}\right\}$ and $w=\left[x_{1}, x_{2}\right]\left[x_{3}, x_{4}\right]$. Then $w^{\prime}=\alpha(w)=w_{1} w_{2}$, with $w_{1}$ and $w_{2}$ both quadratic words as described in Theorem 2.4. Since $w$ is a product of commutators in the hyperbolic Baumslag double $G$ and $\alpha$ is an automorphism, it follows that $w^{\prime}$ is alternating in the same way as $w$ : that is, each $x_{i}$ occurs in $w^{\prime}$ exactly once as $x_{i}$ and exactly once as $x_{i}^{-1}$. Since both $w_{1}$ and $w_{2}$ are quadratic and $F$ is a non-abelian free group, this implies, up to conjugation and renaming, that $w_{1}=[x, y]$ for some $x, y \in F$. Recall that a free group word $[a, b][c, d]$ is not Nielsen equivalent to a word $r^{2} s^{2} t^{2} p^{2}$, otherwise an orientable surface group of genus 2 would be isomorphic to a non-orientable surface group of genus 4 . That this cannot happen is clear from abelianization. Since the amalgamated subgroup $A=\langle W\rangle$ is cyclic and $w_{1}$ is conjugate to an element of $A$, it follows that $[x, y]$ is conjugate to $W^{n}$ for some non-zero $n \in \mathbb{Z}$. However, since a commutator in a free group is never a proper power (see $\left[\mathbf{1 0}\right.$, p. 52] or [4]) this implies that $[x, y]$ is conjugate to $W$ or $W^{-1}$. Since a conjugate of a commutator is also a commutator, it follows that $W=[U, V]$ for some elements $U, V \in F$, proving the theorem in the orientable case.

The proof for the non-orientable case is almost identical except that when we get $w=w_{1} w_{2}$ we must have $w_{1}=x^{2} y^{2}$ for some $x, y \in F$. We must also use the analogous argument that if a free group $F=\langle a, b ;\rangle$ is generated by a system $\left\{r, s, a^{2} b^{2}\right\}$, then there is a free Nielsen transformation from $\left\{r, s, a^{2} b^{2}\right\}$ to $\left\{a, b, a^{2} b^{2}\right\}$ where $a^{2} b^{2}$ is not replaced. As in the orientable case, this follows from Lemma 3.17 in $[\mathbf{1 2}]$ and the remark immediately after that lemma.

We note that a hyperbolic Baumslag double can never contain an orientable surface group of genus 1 (that is, a free abelian group of rank 2) and can never contain a nonorientable surface group of genus less than or equal to 3 by Theorem 2.4.

\section{References}

1. P. Ackermann, B. Fine And G. Rosenberger, Surface groups: motivating examples in combinatorial group theory, in Groups St Andrews 2005, London Mathematical Society Lecture Notes Series, Volume 339, pp. 96-130 (Cambridge University Press, 2007).

2. G. Baumslag, On generalised free products, Math. Z. 78 (1962), 423-438.

3. M. Bestrina And M. Feighn, A combination theorem for negatively curved groups, $J$. Diff. Geom. 35 (1992), 85-101.

4. L. Comerford, C. Edmonds and G. Rosenberger, Commutators as powers in free products, Proc. Am. Math. Soc. 122 (1994), 47-52. 
5. B. Fine, G. Rosenberger and M. Stille, Nielsen transformations and applications: a survey, in Groups Korea 94 (De Gruyter, Berlin, 1995).

6. C. Gordon And H. Wilton, Surface subgroups of doubles of free groups, in preparation.

7. A. Juhasz And G. Rosenberger, On the combinatorial curvature of groups of $F$-type and other one-relator products of cyclics, Contemp. Math. 169 (1994), 373-384.

8. O. Kharlampovich And A. Myasnikov, Hyperbolic groups and free constructions, Trans. Am. Math. Soc. 350(2) (1998), 571-613.

9. S. Kim AND H. Wilton, Surface subgroups of doubles of free groups, in preparation.

10. R. LYNDON AND P. E. SchupP, Combinatorial group theory (Springer, 1977).

11. W. Magnus, A. Karrass And D. Solitar, Combinatorial group theory (Wiley Interscience, 1966).

12. G. Rosenberger, On one-relaor groups that are free products of two free groups with cyclic amalgamation, in Groups St Andrews 1981, pp. 328-344 (Cambridge University Press, 1981).

13. H. Zieschang, Über die Nielsensche Küerzungmethode in freien Produkten mit Amalgam, Invent. Math. 10 (1970), 4-37. 